\title{
Red Blood Cell \\ Structure/Function/Metabolism
}

National Cancer Institute

\section{Source}

National Cancer Institute. Red Blood Cell Structure/Function/Metabolism. NCI Thesaurus.

Code C19446.

Cell biology concerned with structure and function of erythrocytes. 